\title{
Black brane steady states
}

\author{
Irene Amado and Amos Yarom \\ Department of Physics, Technion, \\ Haifa 32000, Israel \\ E-mail: irene.r.amado@gmail.com, ayarom@physics.technion.ac.il
}

ABSTRACT: We follow the evolution of an asymptotically AdS black brane with a fixed temperature gradient at spatial infinity until a steady state is formed. The resulting energy density and energy flux of the steady state in the boundary theory are compared to a conjecture on the behavior of steady states in conformal field theories. Very good agreement is found.

KEYWORDS: Gauge-gravity correspondence, AdS-CFT Correspondence

ARXIV EPRINT: 1501.01627 


\section{Contents}

1 Introduction 1

2 Constructing black brane steady states 3

$\begin{array}{llr}3 & \text { Outlook } & 7\end{array}$

$\begin{array}{lrr}\text { A } & \text { The steady state conjecture } & 8\end{array}$

\section{Introduction}

Predicting the out of equilibrium behavior of many-body systems is a notoriously difficult problem. Many of the tools which have been developed to describe thermodynamic equilibrium are insufficient to explore systems which are far from it. Of the various nonequilibrium phenomenon, one that seems to have a tractable lead is the steady-state problem where one attempts to study the properties of a steady state which emerges once the system is placed between two heat baths. Recent progress on this problem has been seen in a variety of systems which may be described by a quantum field theory, see, e.g., [1-8]. In this work we focus on the steady-state generated when placing a 3 space-time dimensional relativistic conformal gauge-theory between two heat baths.

When placing a system described by a two dimensional conformal field theory between two heat baths its late time, steady state, behavior is completely fixed in terms of the left and right central charges, the levels of the chiral currents (if present) and the relative pressure difference of the heat baths [1-5]. A recent conjecture which appeared in [4-6] attempts to generalize this result to higher dimensional theories.

The form of the conjecture of [4-6] is as follows. Let us consider two heat baths which are infinitely separated along one spatial direction, call it $z$, and are translation invariant along the remaining directions, $x_{\perp}$. Let us denote the pressure of the left and right heat baths by $P_{L}$ and $P_{R}$ respectively, and the relative pressure difference by

$$
\delta p=\frac{P_{L}-P_{R}}{P_{L}+P_{R}}
$$

Without loss of generality we choose $P_{L}>P_{R}$. Consider an initial state whose energy momentum tensor smoothly interpolates between the two heat baths and is also translation invariant along $x_{\perp}$. The mechanism by which energy is propagated in such a setting can be categorized into two types. A flow driven (or ballistic) mechanism by which the energy propagates via sound modes or their non-linear counterpart, and diffusion. If the system is flow driven then we may expect that at late times the energy of the system will propagate with a velocity $v_{L}$ towards the left heat bath and with velocity $v_{R}$ towards the right 

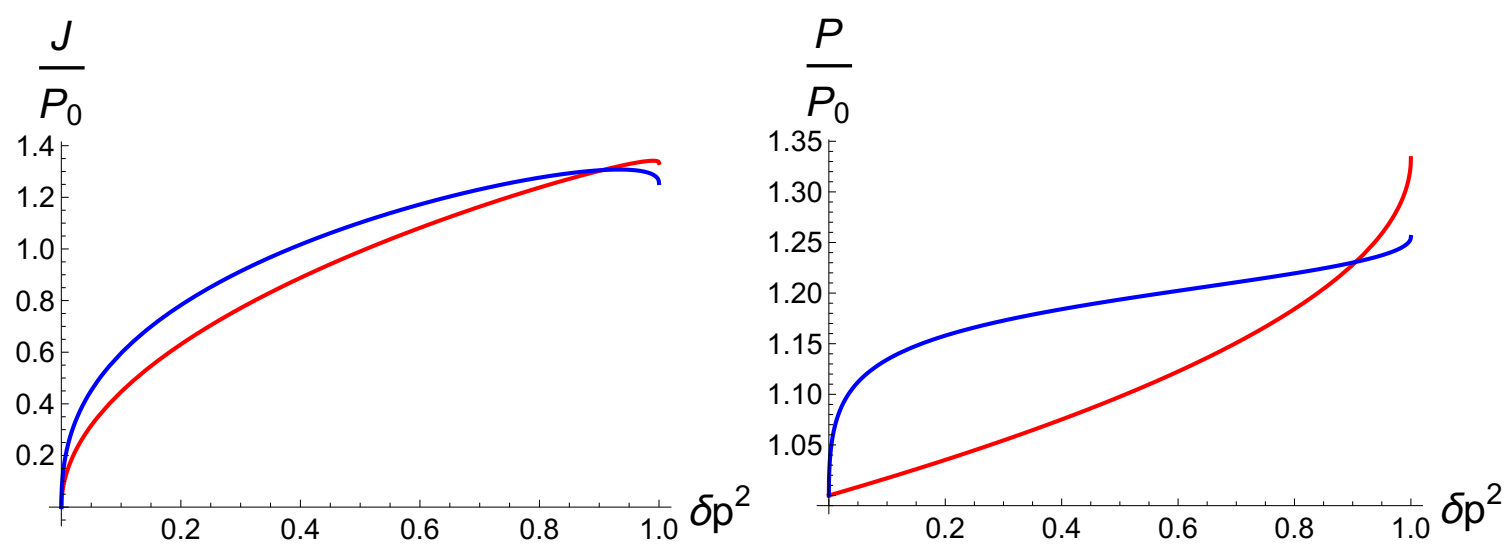

Figure 1. A plot of the conjectured universal values of the late time steady state energy flux (left panel) and pressure (right panel). Here $P_{0}$ is the average pressure of the heat baths, given by (1.3), and $\delta p$ is the relative pressure difference, given by (1.1). The red curves correspond to the thermodynamic branch of steady-states where the expectation value of the energy momentum tensor is equivalent to that of a boosted thermally equilibrated configuration. The blue curve corresponds to the "other" branch of steady-state configurations whose physical role is somewhat obscure.

heat bath generating a steady-state in between. If, in addition, the system thermalizes sufficiently rapidly so that the disturbance immediately behind the propagating waves is in thermal equilibrium, then energy-momentum conservation fixes the expectation value of the pressure, $T^{z z}$, and the energy flux along the $z$ direction, $T^{t z}$, at late times [4-6].

More precisely, in [5] it was shown that the aforementioned physical considerations about the evolution of the system leads to two branches of solutions for the possible late time behavior of $T^{z z}$ and $T^{t z}$. In $[4,6]$ it was argued that a translation invariant steady state allows for only one possible late time solution. The work of $[5]$ and $[4,6]$ are consistent.

In 3 space-time dimensions, the translation invariant steady-state (referred to as the thermodynamic branch in [5]) is characterized by

$$
\begin{aligned}
& \frac{P}{P_{0}} \equiv \lim _{t \rightarrow \infty} \frac{\operatorname{Tr}\left(\rho T^{z z}\right)}{P_{0}}=\frac{1}{3}\left(4-\sqrt{1-\delta p^{2}}\right) \\
& \frac{J}{P_{0}} \equiv \lim _{t \rightarrow \infty} \frac{\operatorname{Tr}\left(\rho T^{t z}\right)}{P_{0}}=\frac{2}{3} \sqrt{5 \delta p^{2}+\sqrt{1-\delta p^{2}}-1}
\end{aligned}
$$

where $P_{0}$ is the average pressure

$$
P_{0}=\frac{1}{2}\left(P_{L}+P_{R}\right)
$$

and $\rho$ represents the density matrix for the steady state. The expressions for $J$ and $P$ in the other branch of the steady state solution is rather long and has been omitted. A plot of $J$ and $P$ as a function of $\delta p$ for both branches appears in figure 1. The reader is referred to [5] for details. A short derivation of the steady state result can be found in appendix A.

The late time steady-state prediction described in (1.2) has been verified explicitly for systems whose time evolution is determined by hydrodynamics [4-6]. While the hydrodynamic description is probably a good one at small relative pressure differences, $\delta p \ll 1$, 
it is unlikely to be valid when the relative pressure difference of the heat baths is large and there are large pressure gradients when one moves away from the steady state towards null infinity. In this work we compare the conjecture of [4-6] to steady states in three dimensional theories which may be described using the AdS/CFT correspondence, up to $\delta p=0.5$, and find excellent agreement.

\section{Constructing black brane steady states}

Simply stated, the AdS/CFT correspondence [9-11], or more generally gauge-gravity duality (see, e.g., [12]), relates a classical $d+1$-dimensional gravitational theory to a planar, $d$-dimensional conformal field theory (CFT) at strong coupling. Of particular importance to the problem at hand is that solutions to classical gravity which follow from the action

$$
S=\frac{1}{2 \kappa^{2}} \int \sqrt{g}\left(R+\frac{6}{L_{\text {AdS }}^{2}}\right) d^{4} x
$$

and asymptote to conformally flat space at spatial infinity hold information about a dual conformal field theory in 3 space-time dimensions.

For instance, the black brane solution to the equations of motion which follow from (2.1) after setting $L_{\mathrm{AdS}}=1$ is given by

$$
d s^{2}=2 d t d r-r^{2}\left(1-\left(\frac{4 \pi T}{3 r}\right)^{3}\right) d t^{2}+r^{2}\left(d x_{\perp}^{2}+d z^{2}\right)
$$

with $T$ the Hawking temperature of the black brane. The solution (2.2) is dual to a thermal state given by a density matrix $\rho=e^{-\beta H}$ of the conformal field theory, with $H$ its Hamiltonian and $\beta=T^{-1}$ its inverse temperature. The expectation value of the energy momentum tensor of the CFT in this thermal state is given by

$$
\operatorname{Tr}\left(\rho T^{\mu \nu}\right)=\left(\begin{array}{ccc}
2 P(T) & 0 & 0 \\
0 & P(T) & 0 \\
0 & 0 & P(T)
\end{array}\right)
$$

with

$$
P(T)=p_{0}\left(\frac{4 \pi T}{3}\right)^{3}
$$

where the value of the dimensionless parameter $p_{0}$ depends on the particular details of the dual theory under consideration. In the (planar and strongly coupled) ABJM theory [13],

$$
p_{0}=\frac{2 N^{2}}{9 \sqrt{2 \lambda}}
$$

with $N$ the rank of the gauge group and $\lambda=N / k$ with $k$ the Chern-Simons level.

In order to generate a steady state in the dual CFT of the type to which the conjecture of [4-6] applies, we consider black branes whose dual energy momentum tensor will satisfy

$$
\lim _{\substack{z \rightarrow \infty \\
t, x_{\perp} \text { finite }}} \operatorname{Tr}\left(\rho T^{\mu \nu}\left(t, x_{\perp}, z\right)\right)=\left(\begin{array}{ccc}
2 P\left(T_{R}\right) & 0 & 0 \\
0 & P\left(T_{R}\right) & 0 \\
0 & 0 & P\left(T_{R}\right)
\end{array}\right)
$$


and

$$
\lim _{\substack{z \rightarrow-\infty \\
t, x_{\perp} \text { finite }}} \operatorname{Tr}\left(\rho T^{\mu \nu}\left(t, x_{\perp}, z\right)\right)=\left(\begin{array}{ccc}
2 P\left(T_{L}\right) & 0 & 0 \\
0 & P\left(T_{L}\right) & 0 \\
0 & 0 & P\left(T_{L}\right)
\end{array}\right)
$$

with $T_{R}$ and $T_{L}$ the temperatures of the right and left heat baths respectively and $\rho$ the density matrix of the system. Geometrically, the conditions (2.6) correspond to a metric which asymptotes to (2.2) as $z$ approaches $\pm \infty$ with corresponding temperature $T_{L}$ and $T_{R}$. An initial configuration for the metric which interpolates between the two asymptotic black brane solutions will evolve in time and a time independent configuration is expected to be generated at late times and finite $z$. Once such a steady state is made available then the energy momentum tensor associated with the late time geometry of such a black brane can be compared to the prediction of [4-6]. This is the strategy we will follow in the remainder of this work. Other examples of out of equilibrium stationary black hole configurations can be found in [14-17].

Consider the metric ansatz

$$
d s^{2}=2 d t(d r-A(t, z, r) d t-F(t, z, r) d z)+\Sigma^{2}(t, z, r)\left(e^{B(t, z, r)} d x_{\perp}^{2}+e^{-B(t, z, r)} d z^{2}\right)
$$

As pointed out in [18] this ansatz is invariant under $r \rightarrow r+\xi(t, z)$. We will fix this residual diffeomorphism symmetry shortly. The equations of motion which follow from (2.1) take the form

$$
\begin{aligned}
4 \partial_{r}^{2} \Sigma+\Sigma\left(\partial_{r} B\right)^{2} & =0 \\
-\Sigma^{2} \partial_{r}^{2} F-\Sigma^{2} \partial_{r} B \partial_{r} F+C_{F}[B, \Sigma] F & =S_{F}[\Sigma, B] \\
4 \Sigma^{3} \partial_{r} \dot{\Sigma}+4 \Sigma^{2} \partial_{r} \Sigma \dot{\Sigma} & =S_{\dot{\Sigma}}[\Sigma, B, F] \\
4 \Sigma^{4} \partial_{r} \dot{B}+4 \Sigma^{3} \partial_{r} \Sigma \dot{B} & =S_{\dot{B}}[\Sigma, B, F, \dot{\Sigma}] \\
2 \Sigma^{4} \partial_{r}^{2} A & =S_{A}[\Sigma, B, F, \dot{\Sigma}, \dot{B}]
\end{aligned}
$$

and

$$
\begin{aligned}
\ddot{\Sigma} & =Q_{\ddot{\Sigma}}[\Sigma, B, F, A] \\
\partial_{z} \dot{F} & =Q_{\partial_{z} \dot{F}}[\Sigma, B, F, A],
\end{aligned}
$$

with the following definitions. Dotted variables are given by

$$
\dot{X}=\partial_{t} X+A \partial_{r} X \quad \ddot{X}=\partial_{t} \dot{X}+A \partial_{r} \dot{X} .
$$

The expressions $C_{F}$ and $S_{X}$ in (2.8) depend only on spatial derivatives of their arguments, and the expressions $Q_{X}$ in (2.9) depend on spatial and time derivative of their arguments.

The asymptotic AdS boundary, located at $r \rightarrow \infty$, is given by the line element

$$
d s^{2}=\left(2 d t d r+r^{2}\left(-d t^{2}+d x_{\perp}^{2}+d z^{2}\right)\right)\left(1+\mathcal{O}\left(r^{-1}\right)\right) .
$$

The energy momentum tensor of the dual theory can be read off of the large $r$ asymptotics of (2.7) as follows. Given (2.11) we find that, under the equations of motion, the large $r$ 
expansion of the metric components $A, F, \Sigma$ and $B$ are given by

$$
\begin{aligned}
& A=\frac{1}{2}(r+\xi(t, z))^{2}-\partial_{t} \xi(t, z)+\frac{a_{1}(t, z)}{r+\xi(t, z)}+\mathcal{O}\left(r^{-2}\right) \\
& F=-\partial_{z} \xi(t, z)+\frac{f_{1}(t, z)}{r+\xi(t, z)}+\frac{3 \partial_{z} b_{3}(t, z)}{4(r+\xi(t, z))^{2}}+\mathcal{O}\left(r^{-3}\right) \\
& \Sigma=r+\xi(t, z)-\frac{3 b_{3}(t, z)}{40(r+\xi(t, z))^{5}}+\mathcal{O}\left(r^{-6}\right) \\
& B=\frac{b_{3}(t, z)}{(r+\xi(t, z))^{3}}+\mathcal{O}\left(r^{-4}\right) .
\end{aligned}
$$

Here $a_{1}(t, z), f_{1}(t, z)$ and $b_{3}(t, z)$ must satisfy the constraints

$$
\partial_{t} a_{1}=\frac{3}{4} \partial_{z} f_{1} \quad \partial_{t} f_{1}=\frac{2}{3} \partial_{z} a_{1}+\partial_{z} b_{3}
$$

(which are a result of (2.9)), but are otherwise undetermined. Once (2.13) are satisfied then (2.8) ensure that (2.9) will hold. Following [19, 20] the energy momentum tensor of the dual theory is given by

$$
\operatorname{Tr}\left(\rho T^{\mu \nu}\right)=p_{0}\left(\begin{array}{ccc}
-2 a_{1} & \frac{3}{2} f_{1} & 0 \\
\frac{3}{2} f_{1} & -a_{1}-\frac{3}{2} b_{3} & 0 \\
0 & 0 & -a_{1}+\frac{3}{2} b_{3}
\end{array}\right),
$$

where $\rho$ is the density matrix associated with the dynamical black brane solution. In order to generate the steady state (2.6) we will impose

$$
p_{0} a_{1}(t, z \rightarrow \infty)=-P\left(T_{R}\right) \quad p_{0} a_{1}(t, z \rightarrow-\infty)=-P\left(T_{L}\right)
$$

and also the initial conditions

$$
f_{1}(t=0, z \rightarrow \pm \infty)=0 \quad b_{3}(t=0, z \rightarrow \pm \infty)=0,
$$

which ensure, by causality, that $f_{1}$ and $b_{3}$ vanish at large $|z|$ for any $t$.

Our strategy for solving (2.8) is identical to that presented in [18]. At $t=0$ we set

$$
B=0 \quad f_{1}=0 \quad a_{1}=-A_{0}\left(1-\alpha \tanh \left(\beta \tanh \left(\frac{z}{\lambda}\right)\right)\right) .
$$

Using (2.17) and fixing the residual diffeomorphism symmetry parameter $\xi(t)$ we can now determine $\Sigma, F, \dot{\Sigma}, \dot{B}$ and $A$ at $t=0$ by solving (2.8) in sequence together with the asymptotic conditions (2.12). From this data, one can determine $B, a_{1}$ and $f_{1}$ at $t=\Delta t$ using (2.10) and (2.13). This procedure can now be repeated to obtain the fields at $t=2 \Delta t$ and so on, ad infinitum.

The numerical scheme which was implemented in order to evolve the metric forward in time was based on pseudo-spectral methods to solve the linear equations (2.8) and 3rd order Adams-Bashforth to evolve forward in time. We used a grid of 23 points along the radial direction, $r$, and 100 points along the $z$ direction which we parametrically compactified using

$$
\zeta=\tanh (z /(\lambda L))
$$



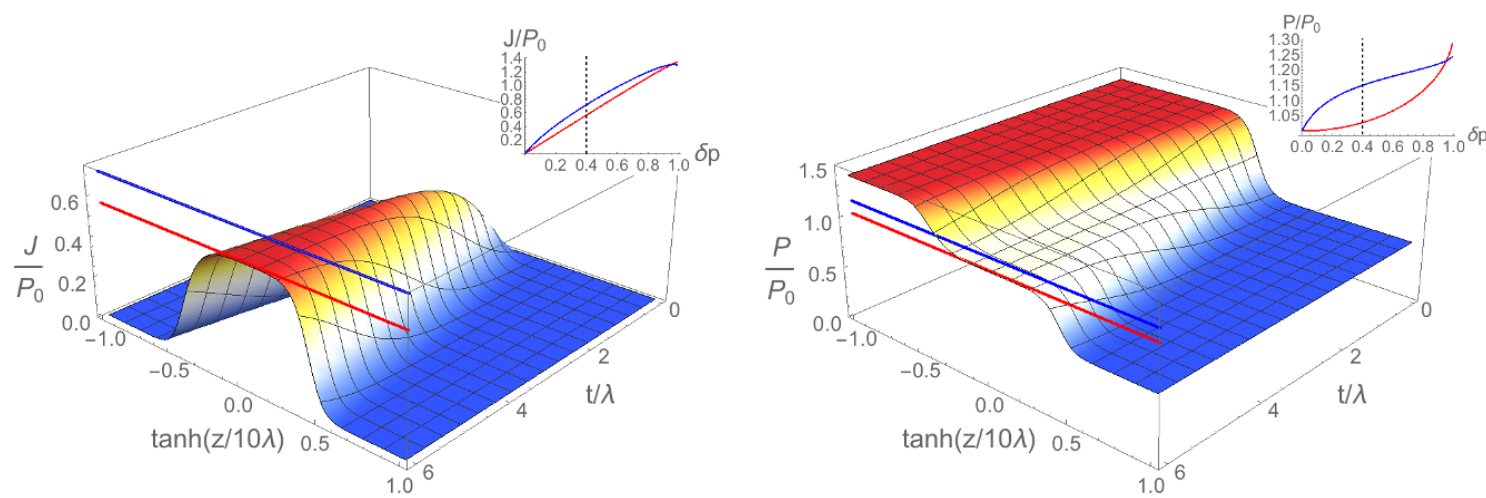

Figure 2. A typical plot of the energy flux (left) and pressure (right) of a theory dual to the black hole described by (2.7). These plots were generated by solving (2.8) with initial conditions given in (2.17) with $\lambda=1, A_{0}=100, \alpha \sim 0.8657$ and $\beta=1 / 2$ (corresponding to $\delta p=0.4$ ). At late times the steady state value of the energy flux and pressure asymptote to the red branch to within $0.2 \%$. The insets are exact copies of the respective panels in figure 1 . The dashed vertical line in the inset is located at $\delta p=0.4$.

for an optimal value of $L$. Rescaling all the boundary coordinates by a factor of $\lambda$ is a symmetry of the equations of motion due to conformal invariance of the boundary theory. In practice we have implemented a code which uses $\lambda=1$. In solving the equations of motion we found it convenient to subtract from $A, \dot{B}, \Sigma, \dot{\Sigma}$ and $F$ the solution to (2.8) at $t=0$ with the initial conditions (2.17) which can be obtained analytically, viz.,

$$
\begin{aligned}
& \Sigma=r+\xi \\
& F=-\partial_{z} \xi \\
& A=\frac{1}{2}(r+\xi)^{2}-\partial_{t} \xi+\frac{a_{1}}{r+\xi} \\
& \dot{\Sigma}=\frac{1}{2}(r+\xi)^{2}+\frac{a_{1}}{r+\xi} \quad \dot{B}=0 .
\end{aligned}
$$

In practice the value of $\xi$ was chosen so that an apparent horizon will be located at $r=1$ which is the endpoint of our grid. We refer the reader to [18] for details of such a scheme.

The final expression for the energy momentum tensor of the dual CFT has been computed using (2.14). We have carried out the computation for several values of $0<\delta p<0.7$. A typical result is plotted in figure 2 . For $\delta p \lesssim 0.5$ we have managed to evolve our numerics for sufficiently long so that the energy flux approached its equilibrium value to an accuracy of less than 1\%. See figure 3 for a fit of the obtained curve from the conjectured expectations. At very large relative pressure difference the black brane configuration at $z \rightarrow \infty$ has a very small temperature relative to the one on at $z \rightarrow-\infty$ and our numerical scheme seems to break down. At best this breakdown is due to the parametric compactification we have used to map the $z$ coordinate to a finite interval. In order to avoid difficulties associated with such compactifications [21], it may be possible to repose the steady state problem using periodic boundary conditions in the $z$ direction. However, if the numerical instability associated with large relative pressure differences is due to the formation of caustics, then one would need a more robust algorithm than that of [18] and summarized here to obtain a handle on the problem. 

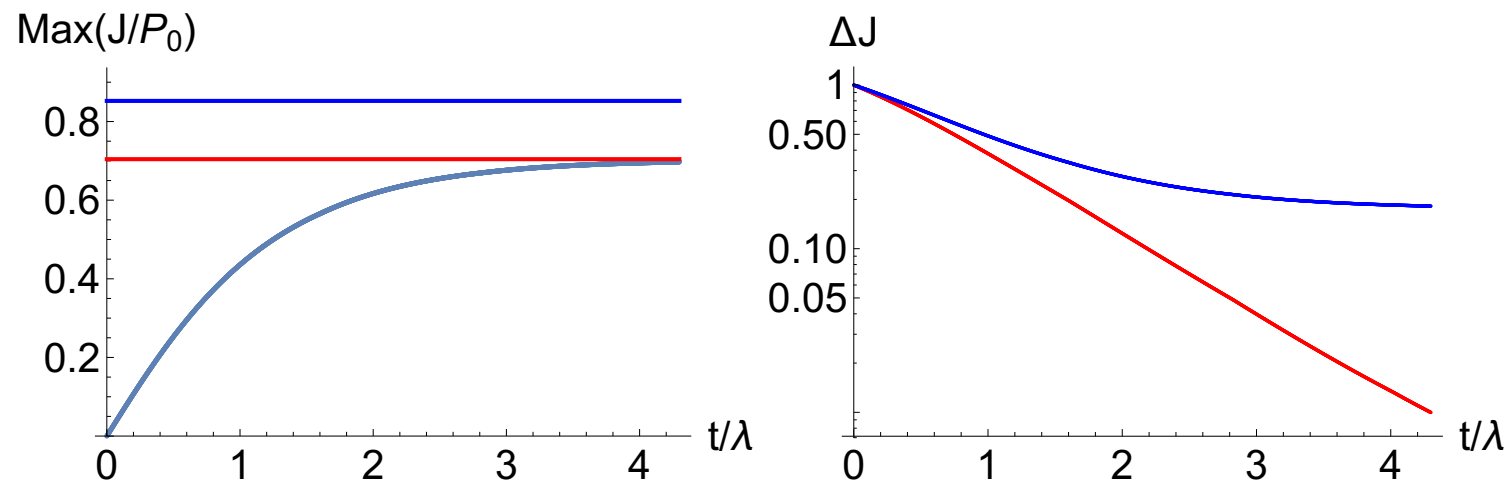

Figure 3. Plots describing the approach to the conjectured steady state configuration. These plots were generated by solving (2.8) with initial conditions given in (2.17) with $\lambda=1, A_{0}=100, \alpha \sim$ 1.082 and $\beta=1 / 2$ (corresponding to $\delta p=0.5$ ). The $z$ coordinate was parametrically compactified as in (2.18) with $L=5$. In the left panel we have plotted, for every time $t$, the maximal (in $z$ ) value for the energy flux $J$ together with the conjectured steady state value of $J$ (red and blue). In the right panel we have plotted the relative difference between the maximal (in $z$ ) value for the energy flux $J$ and the predicted values of the thermodynamic (red) and other (blue) branch.

When $\delta p^{2} \sim 0.9$ the steady state pressure and energy density of the other (blue) branch becomes lower than that of the thermodynamic (red) branch. It would be interesting to check if at such large pressure differences the resulting steady state prefers the other branch.

\section{Outlook}

The duals of asymptotically AdS black branes with a fixed temperature gradient at asymptotic infinity seem to reach a steady state associated with the red branch studied in [4-6] and depicted in figure 1, at least for relatively low pressure differences. When the relative pressure difference between the left and right heat bath was above 0.5 our numerical scheme seemed to break down. The breakdown of our numerical code could be signaling a genuine instability of the black brane steady state, or it could be related to our method of parametrically compactifying spatial infinity. Checking which of the two aforementioned scenarios is correct is of crucial importance in understanding the late time steady state behavior of the system.

A genuine instability of the black brane steady state at large pressure differences could be a signal of a nearby transition between the red and blue branches of solutions. The blue branch steady state is not a boosted thermodynamic equilibrium configuration and its existence provides a marked distinction between the approach of $[4,6]$ and $[5]$ in deriving the steady state.

Another interesting line of research would be to consider charged steady state configurations. The computations of $[22,23]$ seem to indicate that in the presence of conserved charges the universality of (at least) the low pressure behavior of the steady state breaks down. Carrying out a holographic computation with conserved charges may shed light on the possible late time behavior of charged configurations. 


\section{Acknowledgments}

We thank K. Balasubramanian, H.-C. Chang, P. Chesler, A. Karch and K. Schalm for useful discussions and comments. AY and IA are supported by the ISF under grant numbers 495/11, 630/14 and 1981/14, by the BSF under grant number 2014350, by the European commission FP7, under IRG 908049 and by the GIF under grant number 1156/2011.

\section{A The steady state conjecture}

Let us review the conjecture given in [4-6] in detail. In $1+1$ dimensions, the steady state forming in the intermediate region of a CFT connecting two asymptotic heat baths was proven to exist by $[1,2]$. Their result can be rederived by using modest field theory arguments. Consider a two dimensional conformal field theory. In flat space the stress energy tensor is traceless due to conformal invariance, $T_{\mu}^{\mu}=0$. Conservation of the stress energy tensor, $\nabla_{\mu} T^{\mu \nu}=0$, further implies that the energy density and energy flux have to satisfy a wave equation. Thus, the most general form of $T^{\mu \nu}$ is a superposition of a left and a right moving wavefront traveling at the speed of light,

$$
T^{\mu \nu}=\left(\begin{array}{ll}
T_{+}(t+x)+T_{-}(-t+x) & T_{-}(-t+x)-T_{+}(t+x) \\
T_{-}(-t+x)-T_{+}(t+x) & T_{+}(t+x)+T_{-}(-t+x)
\end{array}\right) .
$$

This structure guarantees that the late time behavior will be completely determined by time independent boundary conditions at spatial infinity $x= \pm \infty$. In the case of interest, the system lives between two asymptotic equilibrium heat baths at different pressures, such that

$$
\left.T^{\mu \nu}(t, x)\right|_{x \rightarrow \pm \infty}=\left(\begin{array}{cc}
P_{R / L} & 0 \\
0 & P_{R / L}
\end{array}\right) .
$$

Then, at future infinity we have

$$
\left.T^{\mu \nu}(t, x)\right|_{t \rightarrow \infty}=\left(\begin{array}{cc}
\frac{1}{2}\left(P_{L}+P_{R}\right) & \frac{1}{2}\left(P_{L}-P_{R}\right) \\
\frac{1}{2}\left(P_{L}-P_{R}\right) & \frac{1}{2}\left(P_{L}+P_{R}\right)
\end{array}\right) .
$$

For a finite sized configuration of length $\ell$, we should find an intermediate steady state region and at times $t \gg \ell / c$, the system will be in a steady state fully specified by the asymptotic pressures. The evolution is described by two wavefronts moving in opposite directions at speed of light leaving a universal stationary state in the intermediate region. Notice that this is a flow driven steady state due to the ballistic nature of transport in two dimensional systems. It is also worth noticing that the steady state region is a boosted thermal state.

In order to generalize this results to higher dimensional CFTs, the authors of $[4,6]$ assumed that the putative steady state resulting from thermalization between asymptotic heat baths would still be described by a boosted thermal state. They used gauge/gravity duality to extract the properties of such steady state by noticing that the only regular 
solution dual to a system with a homogeneous constant stress energy tensor is the boosted black brane.

The authors of [5] assumed instead that the evolution of the system would still be described by two wavefronts moving apart at constant velocity and leaving behind a steady state plateau. This approach implicitly assumes flow driven transport where diffusion effects are not dominant. One can then repeat the field theory analysis by imposing suitable boundary conditions in addition to conservation of $T^{\mu \nu}$. At spatial infinity we still have two different heat baths,

$$
T^{\mu \nu}(x \rightarrow \pm \infty)=\left(\begin{array}{cc}
\epsilon\left(P_{0} \mp \Delta P\right) & 0 \\
0 & P_{0} \mp \Delta P
\end{array}\right),
$$

where $\mu, \nu=t, x$ and the remaining components of the stress energy tensor are on the diagonal. At late times the system can be divided into three regions: the region $\mathrm{I},-\infty<$ $x<-L$, described by a left moving wave with velocity $v_{L}$; the region III, $L<x<\infty$, described by a right moving wave with velocity $v_{R}$; and the intermediate region II, $-L<$ $x<L$, corresponding to the steady state. Here $L$ is very large. Therefore, the matching conditions between the different regions read

$$
T_{I}^{\mu \nu}(t \rightarrow \infty)=T_{I I}^{\mu \nu}(x \rightarrow-\infty), \quad T_{I I I}^{\mu \nu}(t \rightarrow \infty)=T_{I I}^{\mu \nu}(x \rightarrow \infty)
$$

and determine the asymptotics of the steady state. To fully determine the stress energy tensor in region II and the velocities $v_{R / L}$ of the wavefronts it is necessary to specify the equation of state $(\epsilon(P)=(d-1) P$ for conformal theories) and also to impose thermal equilibrium in the matching points between the steps and the steady state region. This last condition is less restrictive than the assumption made in $[4,6]$. With these restrictions one can solve the resulting non-linear equations for the pressure and heat current obtaining two branches of solutions. The first solution, which we refer to as the thermodynamic branch, is identical to that of $[4,6]$ and is characterized by

$$
\begin{aligned}
\frac{P}{P_{0}} & =\frac{1}{d}\left(2(d-1)-(d-2) \sqrt{1-\delta p^{2}}\right) \\
\frac{J}{P_{0}} & =\frac{\sqrt{2(d-1)}}{d} \sqrt{\left(d^{2}-2 d+2\right) \delta p^{2}-(d-2)^{2}\left(1-\sqrt{1-\delta p^{2}}\right)}
\end{aligned}
$$

where $\delta p=\Delta P / P_{0}$. This expresion reduces to (A.3) for $d=2$ and to (1.2) for $d=3$. The physical interpretation of the other branch is not yet clear. In particular it is not connected to the $d=2$ solution.

Open Access. This article is distributed under the terms of the Creative Commons Attribution License (CC-BY 4.0), which permits any use, distribution and reproduction in any medium, provided the original author(s) and source are credited.

\section{References}

[1] D. Bernard and B. Doyon, Energy flow in non-equilibrium conformal field theory, J. Phys. A 45 (2012) 362001 [arXiv:1202.0239] [InSPIRE]. 
[2] D. Bernard and B. Doyon, Non-equilibrium steady states in conformal field theory, Annales Henri Poincaré 16 (2014) 113.

[3] D. Bernard and B. Doyon, Time-reversal symmetry and fluctuation relations in non-equilibrium quantum steady states, J. Phys. A 46 (2013) 372001 [arXiv:1306.3900] [INSPIRE].

[4] M.J. Bhaseen, Applications of gauge-gravity duality far from equilibrium, conference talk given at Gauge/gravity duality 2013, July 2013.

[5] H.-C. Chang, A. Karch and A. Yarom, An ansatz for one dimensional steady state configurations, J. Stat. Mech. 1406 (2014) P06018 [arXiv:1311.2590] [INSPIRE].

[6] M.J. Bhaseen, B. Doyon, A. Lucas and K. Schalm, Far from equilibrium energy flow in quantum critical systems, arXiv:1311.3655 [INSPIRE].

[7] M. Collura and G. Martelloni, Non-equilibrium transport in d-dimensional non-interacting fermi gases, J. Stat. Mech. Theor. Exp. 8 (2014) 6 [arXiv:1404.1319].

[8] D. Bernard, B. Doyon and J. Viti, Non-Equilibrium Conformal Field Theories with Impurities, J. Phys. A 48 (2015) 05FT01 [arXiv:1411.0470] [INSPIRE].

[9] J.M. Maldacena, The large $N$ limit of superconformal field theories and supergravity, Int. $J$. Theor. Phys. 38 (1999) 1113 [hep-th/9711200] [INSPIRE].

[10] S.S. Gubser, I.R. Klebanov and A.M. Polyakov, Gauge theory correlators from noncritical string theory, Phys. Lett. B 428 (1998) 105 [hep-th/9802109] [InSPIRE].

[11] E. Witten, Anti-de Sitter space and holography, Adv. Theor. Math. Phys. 2 (1998) 253 [hep-th/9802150] [INSPIRE].

[12] J. McGreevy, Holographic duality with a view toward many-body physics, Adv. High Energy Phys. 2010 (2010) 723105 [arXiv: 0909.0518] [INSPIRE].

[13] O. Aharony, O. Bergman, D.L. Jafferis and J. Maldacena, $N=6$ superconformal Chern-Simons-matter theories, M2-branes and their gravity duals, JHEP 10 (2008) 091 [arXiv: 0806.1218] [INSPIRE].

[14] S. Fischetti and D. Marolf, Flowing Funnels: Heat sources for field theories and the $A d S_{3}$ dual of $\mathrm{CFT}_{2}$ Hawking radiation, Class. Quant. Grav. 29 (2012) 105004 [arXiv:1202.5069] [INSPIRE].

[15] S. Fischetti, D. Marolf and J.E. Santos, AdS flowing black funnels: Stationary AdS black holes with non-Killing horizons and heat transport in the dual CFT, Class. Quant. Grav. 30 (2013) 075001 [arXiv: 1212 .4820] [INSPIRE].

[16] P. Figueras and T. Wiseman, Stationary holographic plasma quenches and numerical methods for non-Killing horizons, Phys. Rev. Lett. 110 (2013) 171602 [arXiv:1212.4498] [INSPIRE].

[17] R. Emparan and M. Martinez, Black String Flow, JHEP 09 (2013) 068 [arXiv:1307.2276] [INSPIRE].

[18] P.M. Chesler and L.G. Yaffe, Numerical solution of gravitational dynamics in asymptotically anti-de Sitter spacetimes, JHEP 07 (2014) 086 [arXiv:1309.1439] [INSPIRE].

[19] V. Balasubramanian and P. Kraus, A stress tensor for Anti-de Sitter gravity, Commun. Math. Phys. 208 (1999) 413 [hep-th/9902121] [InSPIRE]. 
[20] S. de Haro, S.N. Solodukhin and K. Skenderis, Holographic reconstruction of space-time and renormalization in the AdS/CFT correspondence, Commun. Math. Phys. 217 (2001) 595 [hep-th/0002230] [INSPIRE].

[21] J. Boyd, Chebyshev and Fourier Spectral Methods: Second Revised Edition, Dover Books on Mathematics, Dover Publications, (2013).

[22] A. De Luca, G. Martelloni and J. Viti, Stationary states in a free fermionic chain from the quench action method, Phys. Rev. A 91 (2015) 021603 [arXiv:1409.8482].

[23] M. Collura and G. Martelloni, Non-equilibrium transport in d-dimensional non-interacting fermi gases, J. Stat. Mech. Theor. Exp. 8 (2014) 6 [arXiv:1404.1319]. 